\title{
Design and Enactment of Mobile Video Coaching
}

\author{
Sara van der Linden ${ }^{1}$ (D) · Jan van der Meij ${ }^{1} \cdot$ Susan McKenney ${ }^{1}$
}

Published online: 3 August 2019

(C) The Author(s) 2019

\begin{abstract}
Video coaching can powerfully support teacher learning, but its implementation in face-to-face form presents substantial practical challenges. While mobile learning offers several potential solutions, we have limited insight into the design of mobile video coaching programs and especially into how specific technologies can attend to teacher learning needs during video coaching. Using conjecture mapping concepts, this theoretical article presents a generic model for video coaching, and proposes specific design features for mobile video coaching. After distinguishing between technologies that primarily facilitate access and technologies used as cognitive tools, attention is given to (their role in) the design of mobile video coaching in terms of the: activities and tasks planned; materials and resources used; and participation and practices envisioned. Following discussion of mobile video coaching in light of the situated, social, and distributed nature of teacher learning, the article concludes with implications for educational technology coordinators.
\end{abstract}

Keywords Video $\cdot$ Teacher learning $\cdot$ Coaching $\cdot$ Teachers $\cdot$ Mobile learning $\cdot$ Technology $\cdot$ Video coaching $\cdot$ Professional development

\section{Introduction}

Video coaching is an increasingly popular approach to teacher professional development, which has been found to have an effect on both instruction and student outcomes (Kraft et al. 2018). In video coaching, teachers and coaches go through cycles of recording observations and conducting synchronous verbal discussions which are based on the videos. Despite its affordances, the implementation of large-scale in-person teacher coaching programs faces certain challenges, such as high program costs and possible shortage of effective coaches (Kraft et al. 2018).

Technology could potentially mitigate these challenges for a number of reasons. First, transforming video coaching from face-to-face to mobile learning could address the shortage of coaches by enabling them to work regardless of their physical location. This would reduce travel time

Sara van der Linden

sara.vanderlinden@utwente.nl

1 ELAN, Department of Teacher Development, University of Twente, Enschede, the Netherlands and expenses, thus lowering program costs. Second, this could increase accessibility of high-quality coaches. Where teachers in face-to-face video coaching situations are dependent on the coaches that are available in their school or district, mobile video coaching would allow teachers to choose coaches from all over the country. And finally, when teachers are dependent on coaches within schools or district, it is unlikely that there are sufficient coaches available to cater to all teaching domains, e.g. mathematics, biology, language learning etc. Mobile video coaching could offer opportunities to provide coaches with the relevant disciplinary expertise.

Although, mobile technologies offer potential solutions to these challenges, scholarship is lacking on how video coaching could be designed to support both teacher learning and mobility. This theoretical article presents a design for high-quality video coaching and details how technology can support both mobility and important facets of teacher learning. We begin by addressing three questions:

What constitutes high-quality teacher learning? What do we mean by mobile leaning? and What is video coaching? 


\section{Theoretical Framework}

\section{What Constitutes High-Quality Teacher Learning?}

Putnam and Borko (2000) describe teacher learning as situated, social and distributed. These facets of teacher learning are deeply related, and can overlap. As elaborated next, the situated nature speaks to where teachers learn, the social nature speaks to how teachers learn, and the distributed nature speaks to what knowledge, skills or attitudes are learned.

\section{Teacher Learning Is Situated}

Situated perspectives on learning acknowledge that it is not only the learning tasks that influence learning, but that it also encompasses the context, i.e. the interactions and systems in which the learning takes place, making this an integral part of the learning itself (Lave and Wenger 1991). Following this line of reasoning, teacher learning activities cannot be viewed independent from classroom practice. By acknowledging and attending to the context as part of the learning activity itself, it is possible to create knowledge and skills that are directly applicable to practice (Putnam and Borko 2000), therefore heightening relevance and potentially eliciting a sense of agency within teachers. Including authentic tasks is a way to integrate context and relevant support teacher learning (Putnam and Borko 2000). Authentic tasks are learning activities that represent real-world tasks (Brown et al. 1989). As such, they are typically illstructured and often take place in a natural, live settings. Reflection on teaching performance is an authentic task which can be based on first-hand experiences (e.g. viewing videos of one's own classroom practice and reflecting on the action in the video), or second-hand experiences (e.g. viewing videos of other teachers' practice and reflecting on that action).

\section{Teacher Learning Is Social}

If the context is inextricably linked to learning itself, then the social systems in which learning takes place provide the pathways through which teachers learn. Hence, learning does not take place in isolation, but rather "interactions with the people in one's environment are major determinants of both what is learned and how learning takes place." (Putnam and Borko 2000 , p. 5). One common social structure through which interaction and thus learning takes place is the community of practice (Lave and Wenger 1991). While studies of teacher communities show strong affordances (Pareja Roblin et al. 2014), research on teacher discourse within communities of practice clearly show that not all interaction yields teacher learning (Horn et al. 2017). In fact, research points squarely to the importance of support in order to move from more practical conversations to a depth of interaction that stimulates learning (Boschman et al. 2016). Similarly, while videos can present a strong stimulus for discussion and critical reflection on practice, teachers typically require support to focus on the important events or engage in deep reflective talk (Hiebert et al. 2002).

\section{Teacher Learning Is Distributed}

If learning is social and takes place in and through communities, then it follows that knowledge does not reside in only one place or in one person, but is rather distributed amongst people, places and materials (Salomon 1993). Within communities of practice, each participant contributes unique knowledge and beliefs, which together, represent the cognitive resources teachers can draw from to make sense of their practice (Putnam and Borko 2000). This knowledge extends the knowledge of individual teachers but can also be combined. In this view, knowledge is not be seen as a static entity, but rather as something that grows within a community and in the end becomes known to all. This phenomenon has been referred to in the learning sciences as shared cognition. From a perspective of shared understanding, the sum of individual knowledge structures is greater than its parts. Classroom video represents a unique knowledge source that can be used to study classroom practices. Specifically, videos can serve as discussion objects, which prompt participants to share and critique each other's understandings (e.g. about the way students learn or how certain instructional strategies support students). But research shows that expert facilitation is required to bring knowledge sources together, create connections between participants, and assemble or curate materials to support the process (e.g. Van Es and Sherin 2008).

\section{What Do we Mean by Mobile Learning?}

Mobile learning can be understood as a type of learning where learners and the learning itself are not bound to a predetermined physical location (Seppälä and Alamäki 2003). This type of learning has been described in terms as immediate (Kynäslahti 2003), convenient (Kynäslahti 2003) and portable (Seppälä and Alamäki 2003). These attributes of mobile learning can be understood as a need to provide access to learning. In this paper, we also stress the cognitive learning supports that (mobile) technologies can provide.

\section{Access Tools: Learning through and from Technology}

Access to learning relates to permissions, rights, abilities, and means to connect to learning. We distinguish two facets here: Access to expertise and access to materials. Access to expertise means a (technological) connection to actors that can provide (expert) knowledge. Access to expertise can be supported through hardware, software, and infrastructure. For example, portable devices with internet connections are 
needed to connect to wireless networks that facilitate communication. Microphones and cameras can support verbal and non-verbal communication. Access to expertise can also be facilitated through a number of software applications, such as conferencing, messaging or email programs. For verbal communication, an infrastructure is needed with bandwidth that allows for audio conferencing and the addition of nonverbal communication requires bandwidth that supports video conferencing.

Alongside access to expertise, teacher learning requires access to materials, which can be used as input to support teacher development. Next to hardware, such as a portable device and an internet connection, software is needed to (structure permission to) access learning environments. Web authoring, course management applications or shared online storage are examples of this type of software. To access individual file types, different software applications are needed for opening text, image, or video files. On an infrastructure level, bandwidth is needed that allows for streaming video. Data storage requires secure storage facilities, especially when it concerns personal data. Moreover, privacy protocols need to be in place, that explicate the people who have the rights to access the data and the duration it will be stored.

\section{Cognitive Tools: Learning with Technology}

In addition to tools that provide access (to expertise and learning materials), mobile learning can be supported through the use of cognitive tools. Cognitive tools are those that directly assist learners to accomplish cognitive tasks (Lajoie 1993). In the context of mobile learning, where learning is mediated through a handheld device, some software applications serve cognitive tools. This software supports 'learning with technology, instead of learning from technology' (Jonassen and Reeves 1996). Cognitive tools amongst others can be used to (1) aid cognitive processes, such as attention, reflection, problem solving, and metacognition, (2) incorporate activities for learners that would otherwise be inaccessible (Pea 1985). Cognitive tools can take the form of productivity tools, e.g. word processors, file editors, databases, video software, which are normally used for efficiency purposes (Kirschner and Erkens 2006) but have also been shown to enhance learning (Jonassen and Reeves 1996). Hence, individual tools can fulfill multiple functionalities.

\section{What Is Video Coaching?}

Video coaching is a professional development activity whereby teachers and coaches engage in cycles of recording observations and holding video-based synchronous verbal discussions. To be effective, video coaching cycles are ideally implemented repeatedly or combined with other activities that support teacher learning. Video coaching can be aimed at the development of pedagogical knowledge and/ or general teaching skills, such as classroom management (e.g. Early et al. 2017). Alternatively, video coaching can be aimed at the development of pedagogical content knowledge and/ or domainspecific instruction (e.g. Roth et al. 2011). In some cases, video coaching can be aimed at changing beliefs towards teaching and learning (e.g. Meijer et al. 2017). Video coaching attends to situated learning because it includes authentic tasks that integrate classroom practice with professional development. Video coaching attends to social learning because it prompts teachers and coaches to construct and negotiate knowledge through discussions. Video coaching attends to distributed learning because it makes use of different knowledge sources and aims to construct new knowledge that is applicable to practice.

\section{Research Objective and Approach}

Video coaching most often takes place in a face-to-face context (e.g. Roth et al. 2011; Meijer et al. 2017). To our knowledge, there is limited insight into ways technology can facilitate mobile video coaching in environments that attend to the situated, social and distributed nature of teacher learning. Thinking about effective ways to mobilize video coaching, requires looking further than technological solutions alone, to take into consideration how teachers learn through video coaching and the role(s) that (mobile) technologies can play in those processes. Consequently, the objective of this article is to answer the following research question:

How can mobile technologies attend to the situated, social, and distributed nature of teacher learning within the context of high-quality video coaching?

To answer this question, a two-stage literature synthesis was conducted. First, literature on video coaching was reviewed to articulate an evidence-informed theory of action for video coaching in general. Then, literature was reviewed to understand how and to what extent mobile technologies might provide or support the constituent features of video coaching. The results of this two-stage process are given in the following sections.

\section{Stage 1: Video Coaching Theory of Action}

\section{Overall Structure}

Logic models have been recommended as tools for articulating the theory of action underpinning (educational) designs (McKenney and Reeves 2019). Here, we use a specific form of logic modelling, conjecture mapping, because of its explicit 
attention to the argumentative grammar for how learning occurs (Sandoval 2014). As shown in Fig. 1, the conjecture map visualizes a chain of effects.

\section{Design}

The design encompasses all the planned features of the learning environment. These include the activities/ tasks that are planned for learning, the materials/ resources that support the learning processes, and the participation/practices that are envisioned, including the roles and responsibilities of those involved. In video coaching, key activities/tasks include: presentation of information; practicing classroom observation; exploring current practice; setting goals, and designing new teaching and learning experiences. Important materials/ resources for video coaching include tools to activate prior knowledge; examples from practice; and artefacts from practice. Finally, three roles are crucial to the participation/ practices of video coaching: curator; facilitator; coordinator.

\section{Enactment}

Once the learners engage with the learning environment, one speaks of enactment.

While enactment is difficult to capture, the conjecture map portrays it through observable behaviors (reflection on, for, and in action) and artefacts (that support practice), both which can be understood of manifestations of teacher learning.
Reflection on action pertains to discussion in which teachers consider past teaching events. Productive reflection on action is characterized through selective attention, where teachers focus on important actors and events, make productive use of evidence (e.g. use specific video excerpts to support their reasoning about teaching and learning), and demonstrate knowledge-based reasoning (i.e. use of pedagogical, content, or pedagogical content knowledge to interpret the videoed events (Van Es and Sherin 2008). In reflection for action, teachers consider future events. Productive reflection for action is focused on important actors and events in the learning process and involves use of pedagogical, content, or pedagogical content knowledge to reason about steps to be taken and new instructional scenarios. In anticipation of future events, teachers typically create artefacts to support practice. Examples of these products are curriculum materials for teachers and/or students or action plans that detail concrete steps to be taken. Finally, during reflection in action teachers consider their current actions to decide if adjustments are necessary (Schön 1983) while engaging in deliberate practice.

\section{Outcomes and Impact}

Teacher outcomes are produced as a result of the processes during enactment. Teacher outcomes can be understood as cognitive outcomes, i.e. knowledge, behavioral outcomes, i.e. changes in practice, or affective outcomes, i.e. changes in attitudes or beliefs. Finally, it is expected that positive changes

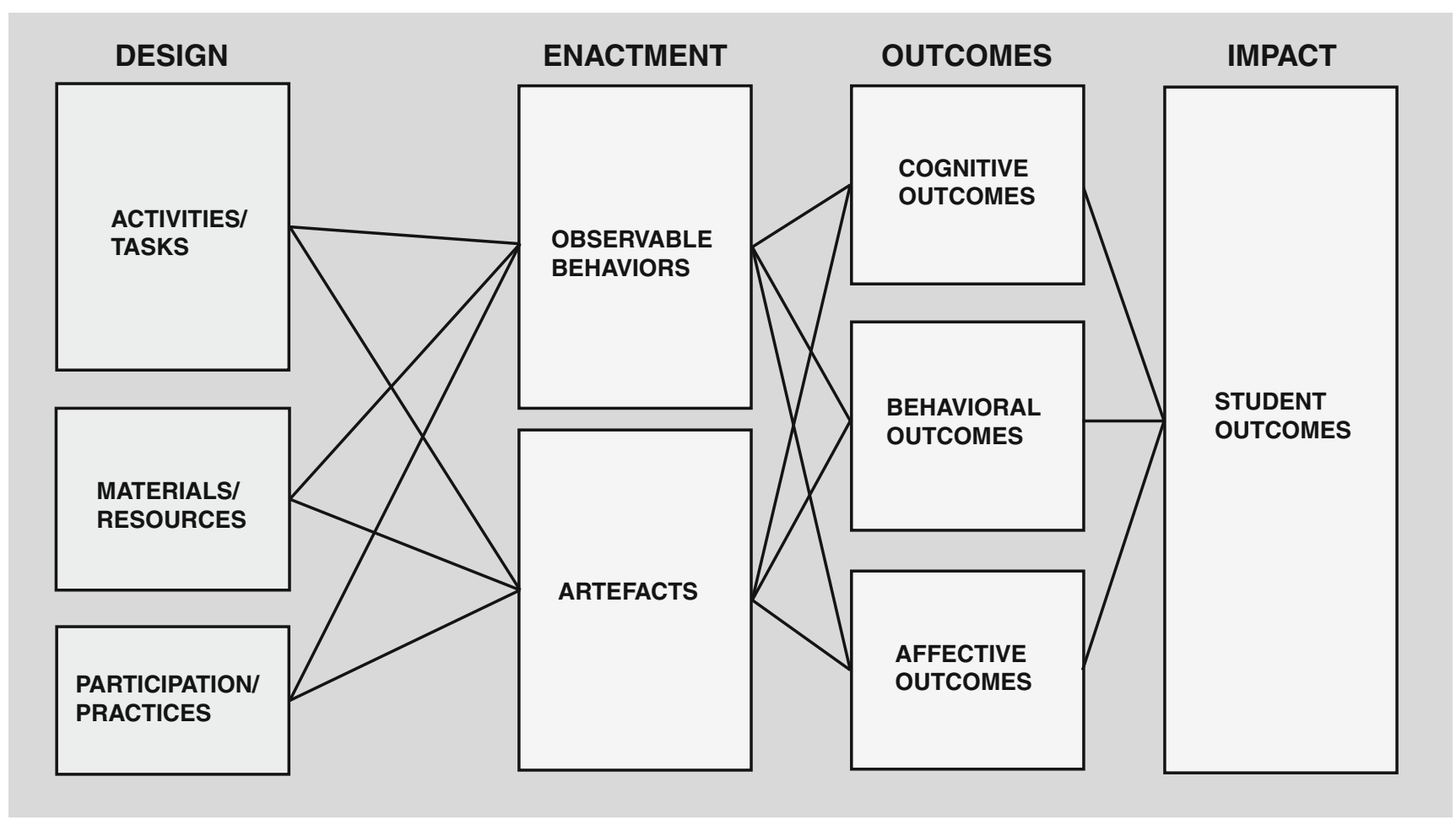

Fig. 1 Generic video coaching model, informed by Sandoval (2014) 
in teacher outcomes elicit impact on student outcomes, such as achievement or engagement.

\section{Stage 2: Mobile Video Coaching Design Guidelines}

As shown above, designing video coaching learning environments requires consideration of activities/ tasks, materials/ resources and participation/ practices in ways that yield reflection in, on and for action as well as artefacts to support practice. This section reports on the second stage of the literature synthesis, and discusses how mobile video coaching could be designed. Following the structure of the detailed conjecture map shown in Fig. 2, this section examines the theory of action behind each design feature, the associated nature of teacher learning, and the role of (mobile) technology to enable or support teacher learning.

\section{Activities/ Tasks}

\section{Presentation of Information Activities}

During presentation of information activities, teachers are provided with access to knowledge. This often happens through, for example, the provision of (online) lectures, provision of readings and/ or collaborative discussion about the readings provided. These activities support conceptual understanding of pedagogical or domain-specific teaching strategies and/ or student learning.

To attend to the social nature of learning, teachers and coaches should critically discuss materials. In mobile video coaching, technology should provide access to expertise through remote interaction, which can be facilitated by cameras and microphones, sufficient bandwidth for audioconferencing and communication software such as audioconferencing apps. To attend to distributed nature of learning this type of activities should incorporate
Fig. 2 Detailed model of video coaching design features and enactment processes

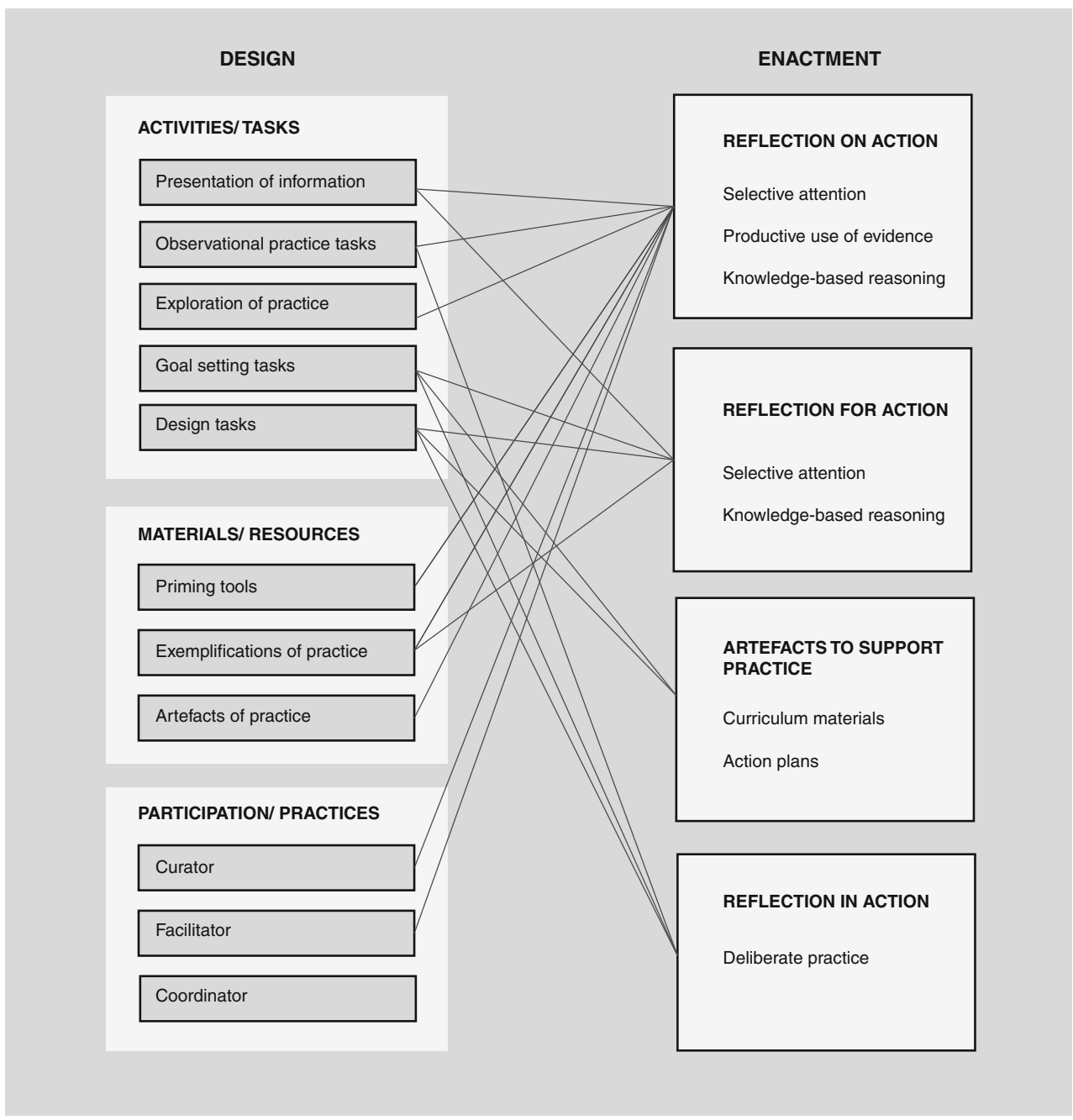


opportunities to share knowledge resources that could contribute to a shared cognition, such as digitalized articles or compiled texts. Technology should provide access to materials and expertise through devices with internet connections, through bandwidth for streaming and through course management software, file readers/ viewers or video players.

\section{Observational Practice Tasks}

During observational practice tasks, teachers rehearse targeted (video analysis) skills, for example through practicing critical video-based discussions and/ or analyzing videos of unknown teacher practice using priming tools. Observational practice tasks are necessary to develop useful lenses for viewing practice. Otherwise, teachers will view classroom video according to lenses that they developed during their study and work (Miller and Zhou 2007).

Learning within observational practice tasks is situated, as teachers engage in reflection on simulated classroom practice, which is represented by unknown teachers video. In mobile video coaching, cognitive tools, such as video players allow teachers to connect with authentic learning contexts, which would otherwise be out of reach. Video annotation tools attend to situated learning by supporting reflective processes (Rich and Hannafin 2009), e.g. by directing teachers attention to specific moments and by prompting them to use evidence from the videos to support their reasoning. To attend to the social nature of learning, teachers and coaches should critically reflect on the simulated practice. In mobile video coaching, this facet of learning can be supported by access to expertise, which can be facilitated through the use of cameras and microphones, audio conferencing software and bandwidth. To attend to the distributed nature of learning, technology should facilitate access to expertise and materials through devices with internet access sufficient bandwidth for streaming.

\section{Exploration of Practice Tasks}

During exploration of practice activities, teachers consider their teaching practice using (pre-recorded videoed) artefacts of practice in a manner can vary from highly structured to fairly unstructured.

The nature of learning within exploration of practice tasks is authentic, as teachers engage in reflection on artefacts from their own practice. In mobile settings, cognitive tools, such as file viewers, video players and video annotation tools, allow teachers to connect with authentic learning contexts. Similar to observational practice tasks, video annotation tools can support reflective processes and reasoning skills (Rich and Hannafin 2009), e.g. by directing attention. The social nature of learning is reflected through interaction between teacher(s) and coach(es) when they critically reflect on artefacts of practice, which should be supported by technology that facilitates distance interaction. In exploration of practice tasks different knowledge sources, e.g. digitalized artefacts and teachers, are needed to share and construct knowledge. In mobile settings technology should provide (secured) access to materials and expertise.

\section{Goal Setting Tasks}

During goal setting activities, teachers and coaches (together) determine the learning objective(s), for example by deciding on the focus of the next planned observation or by creating an action plan. Goal setting activities allow for situated learning as the identification of concrete actions to improve growth areas has a direct relation with and is meant to have an effect on teachers' classroom practice, making it an authentic task.

Goal setting tasks, which requires learners to engage in the metacognitive process of planning the way to approach a task, i.e. to determine which actions to take and set priorities. In mobile video coaching, cognitive tools such as word processing tools can support planning processes, for example by outlining the different steps to be taken. Critical discussions wherein teachers and coaches try to identify which next actions would best support identified growth areas attend to the social nature of learning, although goal setting might as well be an individual activity. Mobile technology supports communication through access to expertise with the help of cameras and microphones, audio conferencing software and bandwidth. Planning next actions often requires consulting different resources for which access to expertise and materials is required, which can be facilitated through technology.

\section{Design Tasks}

During design tasks, teachers arrange, redesign, or create or teaching and learning scenarios, including the tasks, strategies, resources and roles that enable them. Design tasks often follow exploration of practice and or goal setting tasks, where potential growth areas are identified and concrete actions are listed. Design tasks embody opportunities for situated learning, as teachers develop learning scenarios to improve growth areas, which make design activities, participatory authentic tasks.

In mobile video coaching, cognitive tools can support cognitive processes that are important in authentic design tasks, such as creative thinking. Collaborative writing tools can support the visualization of individual ideas, and therefore support processes of idea generation or building on the ideas of others. Attendance to social and distributed nature of learning 
manifests and can be supported through technology in similar ways as in goal setting tasks.

\section{Materials/ Resources}

\section{Priming Tools}

Priming tools help activate prior knowledge and experiences to make connections to (new) conceptual understanding (Windschitl et al. 2012). We use this term in video coaching to refer to text-based resources (digital or printed) that support teachers in recognizing and unpacking specific instructional strategies or aspects of student learning. Use of these tools also supports development of a shared language to communicate about key issues such as learning progressions or observable behaviors. Priming tools provide useful lenses for teachers to review their practice. Varied tools are particularly useful because otherwise, teachers tend to view classroom video (consciously or not) according to the lenses that they developed during their study and work (Miller and Zhou 2007).

Priming tools are inherent cognitive tools because of their function. Priming tools support learning in authentic tasks because they allow teachers to connect specific behaviors to broad conceptualizations of teaching. In addition, these tools support social learning because they help communities to develop a shared language. In mobile video coaching, these tools can be considered artefactual knowledge sources, which need to be made accessible through technology.

\section{Exemplifications of Practice}

Exemplifications of practice are digital or printed materials that demonstrate authentic representations of classroom practice (from classrooms other than those of the participants), often characterized as good or non-examples. These materials can illustrate more or less effective cases of teacher instruction or student learning (within a certain domain). Often videobased exemplifications are used for video coaching activities.

Exemplifications of practice contribute to simulated authentic tasks because these materials illustrate real-life examples. These resources are needed to build shared understandings of effective practices. In mobile video coaching, technology is needed to access these materials and expertise. Technology can facilitate access through devices with internet connections, course management software and file readers or video players that provide access, and bandwidth for streaming as well as secure storage for personal data with privacy protocols in place.

\section{(Videoed) Artefacts of Practice}

(Videoed) Artefacts of practice are physical or digital objects collected from classroom practice, such as videos of teaching practice, audio tapes from practice, student assessments, student work, lesson plans and curriculum materials which are produced by the teacher or the learner. Video-based artefacts of practice are essential components of video coaching and allow teachers to investigate (the effect of instruction on) student learning.

Artefacts of practice that provide an authentic context, can act as a stimulus for discussion (Hiebert et al. 2002), and are resources that hold unique information on classroom practices. In mobile settings, technology is necessary to access the materials and indispensable for the collection process, e.g. recording classroom practice or digitalizing student work. In addition, secure storage space is essential for keeping personal data.

\section{Participation/ Practices}

\section{Curator}

Fulfilling the curator role entails preparing artefacts of practice for use during coaching activities, e.g. editing classroom video into excerpts that illustrate valuable moments of teacher instruction and/ or student learning (in line with the goals of PD); selection of student work to discuss, etc. This role can be fulfilled by the coach or the participants.

In mobile video coaching, cognitive tools can be used to support the cognitive process of organizing, assembling and selecting digital materials, such as file editors, transcription software, video editors and video annotation software by allowing curators to re-structure the materials and help to represent authentic contexts for learning. In addition, technology is needed to access the materials that need curating.

\section{Facilitator}

Fulfilling the facilitator role means directing teachers' attention (e.g. to salient events in a video). It can take place in a variety of ways, e.g. by voicing observations or asking questions and is typically done in a way that aligns with the goals of PD. While the coach does this in most scenarios, participants often co-facilitate in group coaching settings.

By guiding teachers attention to representations of realworld contexts, the facilitator can attend to the situated nature of learning. In mobile video coaching, cognitive tools such as video players and video annotation tools can support attention processes, by highlighting imported events. Often this type of scaffolding takes place during verbal discussions of video, 
attending to the social nature of learning. The facilitator also attends to the notion of distributed learning by making direct use of resources for learning, which are facilitated through technology.

\section{Coordinator}

Those fulfilling the coordinator role organize and communicate about how the coaching will ensue (where, when, with what tools, etc). This is often, but not exclusively, fulfilled by the coach. Even though the role is highly organizational, it is very important for bringing together expertise and materials.

In mobile settings, all communication and the sharing of resources is enacted through technology. Devices with internet connections and software that support communication and the structuring of course materials, i.e. web authoring software or course management software, are needed to support the organization of learning.

\section{Conclusion}

This study reviewed literature to understand how mobile technologies can attend to the situated, social, and distributed nature of teacher learning within the context of high quality video coaching. After distilling a theory of action for video coaching in general (see Figs. 1 and 2), specific design guidelines for mobile video coaching were discussed. Based on that discussion, Table 1 provides an overview of the technologies (specifically, hardware, software and infrastructure) that are essential components mobile video coaching and reveals why, by linking them to the key design features.
The table shows that, in terms of hardware, portable device with internet connections are needed to support all features of the design. In addition, cameras and microphones are most often used communicative and recording functions. In terms of infrastructure, bandwidth is essential for communicative functions in observational practice and exploration of practice tasks. Bandwidth is also essential for distribution and access of video materials. Additionally, secure online storage and privacy protocols are needed to safely store and access (videoed) materials. Finally, software can either be used as an access tool or as a cognitive tool, depending on the function within a design feature.

\section{Discussion}

Mobile technologies can accommodate the situated nature of teacher learning by incorporation of authentic contexts and tasks that would normally be out of reach. Detailed analysis of classroom practice can be facilitated through cognitive tools such as video technology, which not only incorporates an authentic context, but also creates an authentic task. Video software supports detailed analysis by allowing teachers to stop, rewind and replay classroom events over and over. Video technology has the advantage that teachers can view their own classroom and study (the effect of instruction on) student learning, which would be impossible otherwise. Moreover, cognitive tools can support (meta)cognitive processes in ill-defined tasks that have real-world relevance. Video annotation can support reflective processes as it allows teachers to connect tacit knowledge to evidence from the video, therefore allowing often hidden processes of instructional

Table 1 Mobile technology functions in video coaching design features Legend: Grey = Access tools; Hashmarks = Cognitive tools

\begin{tabular}{|c|c|c|c|c|c|c|c|c|c|c|c|c|}
\hline & & \multicolumn{5}{|c|}{ Activities/ tasks } & \multicolumn{3}{|c|}{ Materials/resources } & \multicolumn{3}{|c|}{ Participation/practices } \\
\hline & & $\begin{array}{l}\begin{array}{l}\text { Presentation } \\
\text { of } \\
\text { information }\end{array} \\
\end{array}$ & $\begin{array}{l}\text { Observational } \\
\text { practice tasks }\end{array}$ & $\begin{array}{l}\text { Exploration } \\
\text { of practice }\end{array}$ & $\begin{array}{l}\text { Goal setting } \\
\text { tasks }\end{array}$ & Design tasks & $\begin{array}{l}\text { Priming } \\
\text { tools }\end{array}$ & $\begin{array}{l}\text { Exemplifications } \\
\text { of practice }\end{array}$ & $\begin{array}{l}\text { (Videoed) } \\
\text { Artefacts } \\
\text { of practice }\end{array}$ & Curator & Facilitator & Coordinator \\
\hline \multirow{3}{*}{ 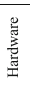 } & Portable device & & & & & & & & & & & \\
\hline & with internet connection & & & & & & & & & & & \\
\hline & with camera and microphone. & & & & & & & & & & & \\
\hline \multirow{18}{*}{ 总 } & Audio (video) conferencing & & & & & & & & & & & \\
\hline & Messaging & & & & & & & & & & & \\
\hline & Email & & & & & & & & & & & \\
\hline & Web authoring software & & & & & & & & & & & \\
\hline & $\begin{array}{l}\text { Project/ course management } \\
\text { tools }\end{array}$ & & & & & & & & & & & \\
\hline & File reader/viewer & & & & & & & & & & & \\
\hline & File writer & & & & & & & & & & & \\
\hline & File editor & & & & & & & & & & & \\
\hline & Transcription software & & & & & & & & & & & \\
\hline & Video player & & & & & & & & & & & \\
\hline & Video editor & & & & & & & & & & & \\
\hline & Video annotation software & & & & & & & & & & & \\
\hline & Video recording software & & & & & & & & & & & \\
\hline & $\begin{array}{l}\text { Visualization tools } \\
\end{array}$ & & & & & & & & & & & \\
\hline & Planning tools & & & & & & & & & & & \\
\hline & Collaborative writing tools & & & & & & & & & & & \\
\hline & Collaborative planning tools & & & & & & & & & & & \\
\hline & Shared online storage space & & & & & & & & & & & \\
\hline \multirow{5}{*}{ 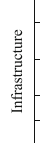 } & Bandwidth for streaming & & & & & & & & & & & \\
\hline & $\begin{array}{l}\text { Bandwidth for audio/video } \\
\text { conferencing }\end{array}$ & & & & & & & & & & & \\
\hline & $\begin{array}{l}\begin{array}{l}\text { Secure storage (online or } \\
\text { offline) }\end{array} \\
\text { (a) }\end{array}$ & & & & & & & & & & & \\
\hline & Secure storage (online) & & & & & & & & & & & \\
\hline & Privacy protocols for data & & & & & & & & & & & \\
\hline
\end{tabular}


decision making to surface (Rich and Hannafin 2009). In addition, video annotation can support teachers reasoning skills when frameworks are incorporated, because teachers are asked to apply the frameworks or lenses to the practice in the video, therefore connecting abstract concepts to instructional behaviors (Sherin and van Es 2005). Further, technology can provide access to expert performance, which is an important aspect of an authentic context (Herrington and Parker 2013). These simulated contexts provide opportunities to watch experts perform tasks while not being present. Video of expert performance or exemplifications of practice can illustrate how to perform instructional behavior, therefore acting as a form of modelling, which is commonly associated with coaching.

Mobile tools that provide access to expertise and support cognition can attend to the social nature of teacher learning. If social learning is perceived as learning that occurs through interaction in communities of practice, then tools are needed to make interaction possible between remote actors. Communication tools can provide access to expertise through use of audio or video conferencing software. In addition, the infrastructure and hardware that permits verbal interaction from a distance needs to be in place. Technology can facilitate (inter) institutional communities of practice, but the implementation of technology that facilitates communication between distant actors does not guarantee learning. The question remains if and how technology can support verbal reflective discussions. It appears that a facilitator or coach is still important in facilitating these processes (e.g. Hiebert et al. 2002).

Mobile technologies support the distributed nature of teacher learning through access to expertise and materials. Technology allows access to materials which are indispensable for teacher learning. Through technology, teachers can share resources and provide access to their classrooms. As such, videoed classrooms can now act as knowledge sources that can be studied and used to construct new knowledge. This is important for teacher learning, because teaching is an isolated profession where these contexts would normally stay hidden, due to practical constraints (Zhang et al. 2011). Other teachers' video provides a window into practice that allows teachers to compare their own teaching to their peers.

\section{Implications}

Mobile video coaching can offer many affordances for practice. This article shows that technology can support access (to materials and expertise) as well as cognition, both of which are important aspects of mobile learning. However, the technology itself does not guarantee learning. The tools mentioned in this article are all productivity tools and therefore easily accessible. However, these tools should be considered dumb tools, which means that the way these tools are used determines in what way and to what extend they can support learning. Before thinking about the technology, the learning objectives should be clarified for different activities, where after technology can be fitted to these objectives.

We close by noting that technology coordinators have an important role to play in mobile video coaching. They can help coaches think about ways teachers can learn from, through, and with technology; they can also co-design learning environments. But if educational technology coordinators need to fulfill these roles, they need to have awareness of the functional opportunities that the different tools have to offer, instead of only focusing on the technical specifications. The theory of action the design guidelines provided here articulate important considerations for all of those who would endeavor to realize mobile video coaching for teacher professional development.

Funding This research was supported by a grant from the Dutch Ministry of Education, Culture and Science as part of the project Begeleiding Startende Leraren, Meesterschap Oost, project number BSL2013-06.

\section{Compliance with Ethical Standards}

Ethical Approval This article does not contain any studies with human participants or animals performed by any of the authors.

Conflict of Interest The authors declare that they have no conflict of interest.

Open Access This article is distributed under the terms of the Creative Commons Attribution 4.0 International License (http:// creativecommons.org/licenses/by/4.0/), which permits unrestricted use, distribution, and reproduction in any medium, provided you give appropriate credit to the original author(s) and the source, provide a link to the Creative Commons license, and indicate if changes were made.

\section{References}

Boschman, F., McKenney, S., Pieters, J., \& Voogt, J. (2016). Exploring the role of content knowledge in teacher design conversations. Journal of Computer Assisted Learning, 3(2), 157-169.

Brown, J. S., Collins, A., \& Duguid, P. (1989). Situated cognition and the culture of learning. Educational Researcher, 18(1), 32-42.

Early, D. M., Maxwell, K. L., Ponder, B. D., \& Pan, Y. (2017). Improving teacher-child interactions: a randomized controlled trial of making the Most of classroom interactions and my teaching partner professional development models. Early Childhood Research Quarterly, $38,57-70$.

Herrington, J., \& Parker, J. (2013). Emerging technologies as cognitive tools for authentic learning. British Journal of Educational Technology, 44(4), 607-615.

Hiebert, J., Gallimore, R., \& Stigler, J. W. (2002). A knowledge base for the teaching profession: what would it look like and how can we get one? Educational Researcher, 31(5), 3-15. 
Horn, I. S., Garner, B., Kane, B. D., \& Brasel, J. (2017). A taxonomy of instructional learning opportunities in teachers' workgroup conversations. Journal of Teacher Education, 68(1), 41-54.

Jonassen, D. H., \& Reeves, T. C. (1996). Learning with technology: Using computers as cognitive tools. In D. H. Jonassen (Ed.), Handbook of research for educational communications and technology (pp. 693-719). New York: Macmillan.

Kirschner, P. A., \& Erkens, G. (2006). Cognitive tools and mindtools for collaborative learning. Journal of Educational Computing Research, 35(2), 199-209.

Kraft, M. A., Blazar, D., \& Hogan, D. (2018). The effect of teacher coaching on instruction and achievement: a meta-analysis of the causal evidence. Review of Educational Research, 88(4), 547-588.

Kynäslahti, H. (2003). In search of elements of mobility in the context of education. In H. Kynäslahti \& P. Seppälä (Eds.), Mobile learning (pp. 41-48). Helsinki: IT Press.

Lajoie, S. P. (1993). Computer environments as cognitive tools for enhancing learning. In S. P. Lajoie \& S. J. Derry (Eds.), Computers as cognitive tools (pp. 261-288). New York: Routledge.

Lave, J., \& Wenger, E. (1991). Situated learning: Legitimate peripheral participation. Cambridge: Cambridge university press.

McKenney, S., \& Reeves, T. C. (2019). Conducting educational design research. Abingdon: Routledge.

Meijer, M. J., Kuijpers, M., Boei, F., Vrieling, E., \& Geijsel, F. (2017). Professional development of teacher-educators towards transformative learning. Professional Development in Education, 43(5), 819840.

Miller, K., \& Zhou, X. (2007). Learning from classroom video: what makes it compelling and what makes it hard. In R. Goldman, R. Pea, B. Barron, \& S. J. Derry (Eds.). Video research in the learning sciences. (pp 321-334). New York: Routledge.

Pareja Roblin, N., Ormel, B., McKenney, S., Voogt, J., \& Pieters, J. (2014). Linking research and practice through teacher communities: a place where formal and practical knowledge meet? European Journal of Teacher Education, 37(2), 183-203.

Pea, R. D. (1985). Beyond amplification: using the computer to reorganize mental functioning. Educational Psychologist, 20(4), 167-182.
Putnam, R. T., \& Borko, H. (2000). What do new views of knowledge and thinking have to say about research on teacher learning? Educational Researcher, 29(1), 4-15.

Rich, P. J., \& Hannafin, M. (2009). Video annotation tools: technologies to scaffold, structure, and transform teacher reflection. Journal of Teacher Education, 60(1), 52-67.

Roth, K. J., Garnier, H. E., Chen, C., Lemmens, M., Schwille, K., \& Wickler, N. I. (2011). Videobased lesson analysis: effective science PD for teacher and student learning. Journal of Research in Science Teaching, 48(2), 117-148.

Salomon, G. (Ed.). (1993). Distributed cognitions: Psychological and educational considerations. Cambridge: Cambridge University Press.

Sandoval, W. (2014). Conjecture mapping: an approach to systematic educational design research. Journal of the Learning Sciences, 23(1), 18-36.

Schön, D. (1983). The reflective practitioner. How professionals think in action. New York: Basic Books.

Seppälä, P., \& Alamäki, H. (2003). Mobile learning in teacher training. Journal of Computer Assisted Learning, 19(3), 330-335.

Sherin, M. G., \& van Es, E. A. (2005). Using video to support teachers' ability to notice classroom interactions. Journal of Technology and Teacher Education, 13(3), 475-491.

Van Es, E. A., \& Sherin, M. G. (2008). Mathematics teachers' "learning to notice" in the context of a video club. Teaching and Teacher Education, 24(2), 244-276.

Windschitl, M., Thompson, J., Braaten, M., \& Stroupe, D. (2012). Proposing a core set of instructional practices and tools for teachers of science. Science Education, 96(5), 878-903.

Zhang, M., Lundeberg, M., Koehler, M. J., \& Eberhardt, J. (2011). Understanding affordances and challenges of three types of video for teacher professional development. Teaching and Teacher Education, 27(2), 454-462.

Publisher's Note Springer Nature remains neutral with regard to jurisdictional claims in published maps and institutional affiliations. 